\title{
Contribution à la connaissance des eaux souterraines du Danube à Vienne
}

\author{
S. Negreal \\ P. Pospisil ${ }^{2}$
}

Mots-clés : Cladocera, eaux souterraines, Danube, Lobau.

Fondés sur le matériel recueilli pendant deux campagnes de recherches (1971-1980 et 1991-1993) dans les eaux souterraines du Danube à Vienne (zone Lobau), les auteurs font un bilan des Cladocères récoltés dans ce milieu. Parmi les 16 espèces inventoriées, 5 sont stygophiles : Alonella nana, Chydorus sphaericus, Alona guttata, Acroperus harpae et Biapertura affinis. Toutes les données montrent que les espèces benthiques des eaux souterraines du Lobau sont en liaison étroite avec celles de la surface (bras mort Eberschüttwasser), formant des populations communes «nappe alluviale-benthos» dont la dynamique est fortement influencée par le régime hydrologique.

\section{A contribution to information on the ground water of the Danube near Vienna}

Keywords : Cladocera, ground water, Danube, Lobau.

Cladocera were recorded from samples collected over two periods (1971-1980 and 1991-1993) from the ground water of the Danube near Vienna (Lobau zone). Of the 16 species recorded, 5 are stygophiles: Alonella nana, Chydorus sphaericus, Alona guttata, Acroperus harpae and Biapertura affinis. All the data show that the benthic species of the ground water at Lobau are closely connected with species at the surface (the stagnant arm Ebertschüttwasser) and form the community of the «alluvial-benthic stratum», the dynamics of which are strongly influenced by the hydrological conditions.

\section{Introduction}

Les eaux souterraines de la région de Vienne font l'objet de recherches écologiques intensives depuis plusieurs années (Danielopol 1976 a, b, c, 1983, 1984, 1989, 1991, Danielopol et al. 1992, Dreher et al. 1994, Haase 1993, Klausnitzer \& Pospisil 1991, Pospisil 1989, 1994 a, b, Pospisil \& Danielopol 1990, Rogulj et al. 1993). La plupart des recherches ont été effectuées dans la zone inondable du Danube, à Lobau. C'est un secteur de la terrasse inférieure rive gauche

1. Institut de Spéologie «Émile Racovitza», str. Frumoasa 11, Bucuresti 78114, Roumanie.

2. Limnologisches Institut, österreichische Akademie der Wissenschaften, A-5310 Mondsee, Autriche. du fleuve, située au sud-est de Vienne, à $150 \mathrm{~m}$ d'altitude. Quoique l'eau souterraine de ce-secteur soit caractérisée fréquemment par l'hypoxie, la présence d'une riche faune composée d'éléments hypogés et épigés a été constatée (Danielopol 1983, 1991, Danielopol et al. 1992, Mosslacher 1994, Pospisil 1989, 1994 a, b). Les recherches effectuées dans les eaux souterraines de deux zones situées à côté du bras mort Eberschüttwasser (Fig. 1, A et C) ont entre autres, porté sur les Cladocères.

Dans ce travail, basé sur le matériel recueilli pendant deux périodes (1979-1981 et 1991-1993), nous faisons un bilan des résultats sur les Cladocères souterrains des deux zones mentionnées qui contribuera à la connaissance des Cladocères des eaux épigées et souterraines des bras morts du Danube. 


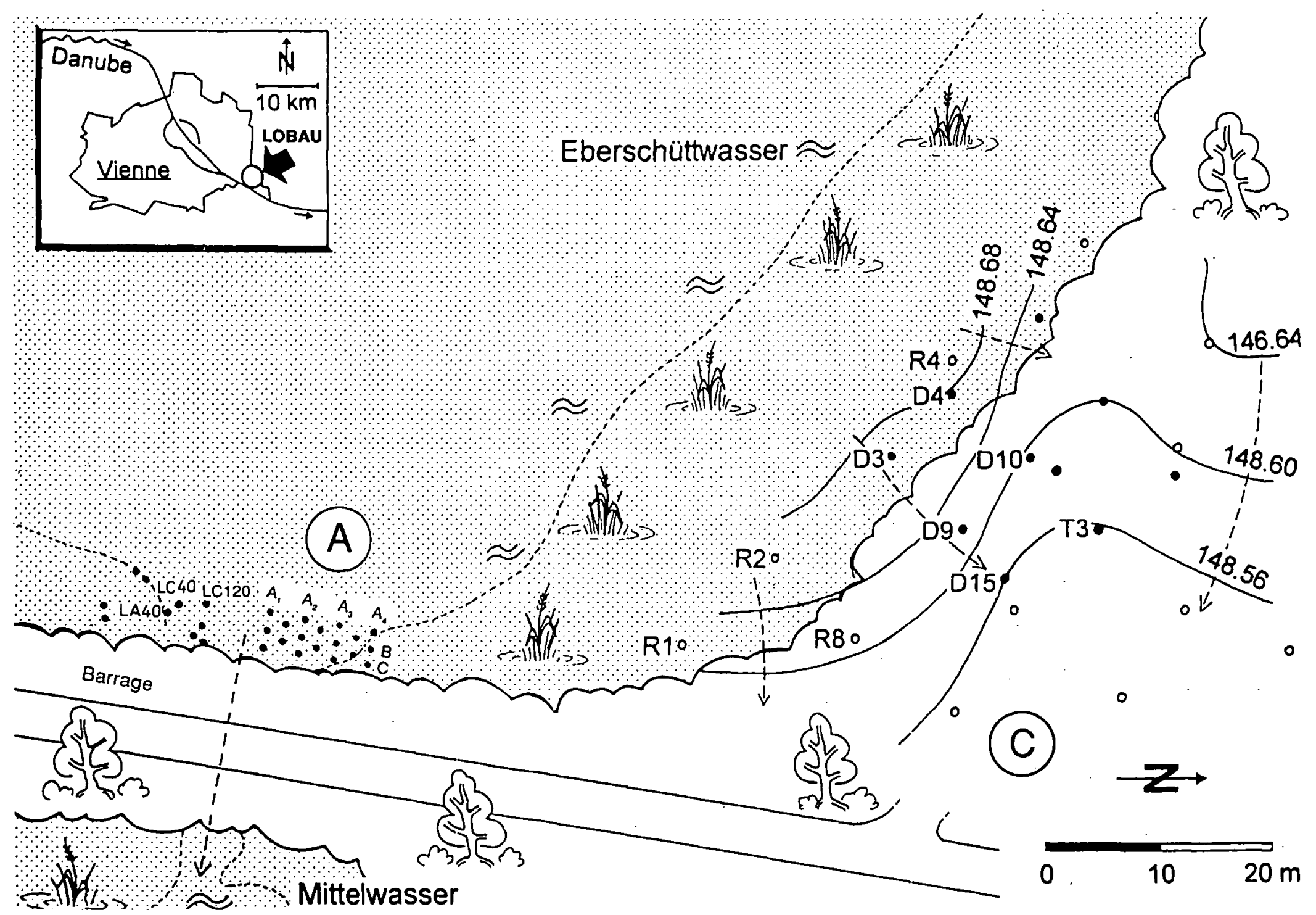

Fig. 1. Stations à Cladocères dans les eaux souterraines du Lobau à Vienne.

A et $C$ : zones qui ont fait l'objet de recherches écologiques et faunistiques suivies (voir texte). Les symboles A, B, C, D, E, L, R, T indiquent les stations positives pour les Cladocères. Les stations négatives sont figurées sans symboles. Les lignes continues désignent les courbes de niveau en surface (mètres au-dessus de l'Adriatique). La ligne pointillée sépare l'eau libre du bras mort Eberschüttwasser de la zone à roseaux. Les flèches en pointillé indiquent la direction de la circulation des eaux souterraines pendant un jour typique (15.1.1992). En blanc, la zone terrestre proprement dite. Un barrage de terre, construit le siècle dernier, sépare les bras morts Eberschüttwasser et Mittelwasser. Pour d'autres informations hydrologiques, voir Dreher et al. (1995) (Original, Pospisil del.).

Fig. 1. Sites with Cladocera in the ground water of Lobau near Vienna.

$A$ and $C$ : zones where ecological and faunistic research was performed (see text). The symbols A, B, C, D, E, L, R, T indicate the positive sites for Cladocera. Negative sites are shown without symbols. The continuous line shows the surface water level (metres above the Adriatic). The dotted line separates the open water of the stagnant arm Ebertschüttwasser from the reed zone. The dotted arrows indicate the direction of underground water circulation during a typical day (15 January 1992). The terrestrial zone is shown in white. An earth dam, built in the last century, separates the stagnant arms Ebertschüttwasser and Mittelwasser. For other hydrological information, see Dreher et al. (1995) (Original, Pospisil del.).

\section{Méthodes. Stations à Cladocères. Abré- viations}

\subsection{Méthodes utilisées dans l'étude de l'écosystè- me de Lobau}

La plupart des bras morts du secteur Lobau sont isolés du chenal du Danube par des digues construites il y a 120 ans. Ceux-ci sont pourtant alimentés par les eaux souterraines, dont le régime est déterminé par le Danube. Les deux zones où l'on a trouvé des Cladocères (A et $C$ ) sont situées dans le périmètre du bras mort Eber- 
schüttwasser, à environ $1 \mathrm{~km}$ du chenal du Danube (Fig. 1).

La zone A, a comme substratum un sédiment formé par du sable et du gravier et est en permanence couverte par l'eau du bras mort Eberschüttwasser. Les recherches effectuées dans cette zone ont été menées de 1979 à 1981, auxquelles s'ajoutent les observations de Danielopol $(1983,1991)$. Les sondes piézométriques en métal utilisées (LA, LB ...) ont $3,5 \mathrm{~cm}$ de diamètre et sont perforées sur les derniers 10 centimètres. $A$ l'aide d'une pompe Bou-Rouch, périodiquement 3 litres d'eau souterraine de chaque sonde ont été filtrés. Les échantillons ainsi prélevés et fixés au formol, ont été filtrés sur tamis de $100 \mu \mathrm{m}$ de vide de maille. Ensuite, les animaux ont été séparés du sédiment par trois agitations et décantations successives, triés au stéréomicroscope et conservés dans l' alcool.

La zone $\mathrm{C}$ est située au bord du bras mort Eberschüttwasser, près de la lisière de roseau. Il s'agit d'une partie de la zone inondable du Danube couverte d'une forêt de peupliers (Fig. 1). Les sondes ont été placées jusqu'à $30 \mathrm{~m}$ de distance du bord du bras mort qui est très variable. Les recherches dans cette zone ont été menées de 1991 à 1993, outre les travaux de Danielopol et al. (1992) et Pospisil (1994 b). Les sondes piézométriques en plexiglas (D3, D4...) ou en métal (T3) utilisées ont $5 \mathrm{~cm}$ de diamètre et sont perforées sur toute leur longueur. A l'aide d'une pompe Bou-Rouch à «Doppelpacker» (doubles manchettes isolantes distales) (Danielopol \& Niederreiter 1987), périodiquement, 5 litres d'eau souterraine de chaque sonde ont été prélevés, filtrés au tamis de $100 \mu \mathrm{m}$ de vide de maille avant fixation au formol et coloration au Rose-Bengale. Par la suite, les échantillons ont été lavés, décantés plusieurs fois, triés au stéréomicroscope et conservés au formol. Les sondes piézométriques en plexiglas $\mathrm{R} 1, \mathrm{R} 2 \ldots$ ont $2,5 \mathrm{~cm}$ de diamètre et sont perforées sur les derniers 10 centimètres. A partir de ces sondes on a également filtré, périodiquement, 5 litres d'eau souterraine à l'aide d'une pompe Bou-Rouch.

\subsection{Caractéristiques des zones à Cladocères (A et} C)

La zone A (Fig. 1), est située dans le bras mort mésotrophe Eberschüttwasser. Ce dernier subit des oscillations du niveau de l'eau. A une profondeur de 0,4$0,5 \mathrm{~m}$ le sédiment est composé de gravier, de sable, de sédiment limoneux et de détritus et la quantité des éléments fins diminue à une profondeur de 3-4 m (Danielopol 1983, 1991). L'eau de surface pénètre par la digue en terre et apparaît de nouveau, de l'autre côté, dans le bras mort Mittelwasser. Pendant les périodes de haut niveau de l'eau, la direction du courant peut être inversée temporairement (Dreher et al. 1994, Pospisil 1994 a, b). Cet «effet de lavage» est probablement la cause du maintien de la perméabilité pour une période plus longue. La concentration en oxygène à - $0,5 \mathrm{~m}$ de profondeur, moindre qu'à la surface, est généralement supérieure à $1 \mathrm{mg} . \mathrm{l}^{-1}$ (Danielopol 1981, 1991) ; à - 1,2 m elle est évidemment diminuée. En ce qui concerne la température du sédiment, à- $0,5 \mathrm{~m}$ de profondeur, les importantes variations thermiques (entre 5 et $22^{\circ} \mathrm{C}$ ) soulignent l'influence dominante de l'eau de surface. A plus de $4 \mathrm{~m}$ de profondeur, les variations se situent entre 7 et $14,5^{\circ} \mathrm{C}$. L'amplitude des valeurs de l'oxygène et de la température baissent donc en fonction de la profondeur, ce qui est généralement le cas pour les eaux souterraines.

La zone $\mathrm{C}$, contrairement à la zone $\mathrm{A}, \mathrm{n}$ 'est inondée que lors des grandes crues du Danube et pendant des périodes plus brèves. En pareil cas, du fait de la position des sondes à la ligne moyenne du bord (qui correspond approximativement à la limite roseau-forêt) seules les sondes D3, D4, D5, R1, R2, R4 et R6 (Fig. 1), peuvent être couvertes par l'eau pendant plusieurs jours. Une mini-vidéocaméra dans une boite étanche (Niederreiter \& Danielopol 1991) et des sondes en plexiglas ont permis d'établir les profils donnant des informations sur la granulométrie ainsi que sur les couches du sédiment jusqu'à $10 \mathrm{~m}$ de profondeur (Pospisil $1994 \mathrm{a}, \mathrm{b}$ ). On a ainsi constaté que le sédiment est du gravier grossier et qu'il contient en proportions différentes du sable et du sédiment limoneux. Dans le cas des sondes D3, D4, D5 et D9 on observe la présence d'une couche située près de la surface dont l'espace interstitiel est presque vide, permettant la libre circulation de l'eau. D'après Dreher et al. (1995), les propriétés de l'eau souterraine de ce site sont dominées par la dynamique de l'eau du bras mort Eberschüttwasser et celle du Danube. A haut niveau, surtout au printemps, l'eau pénètre plus profondément dans le sédiment, rapprochant les valeurs des températures de l'eau souterraine de celles de surface et déterminant des concentrations en oxygène plus fortes dans l'eau souterraine. Au contraire, à bas niveau, surtout durant la période chaude estivale, l'infiltration de l'eau de surface diminue, la consommation de l'oxygène s'accroît, occasionnant de fréquentes conditions d'hypoxie $\left(\mathrm{O}_{2}<0,5\right.$

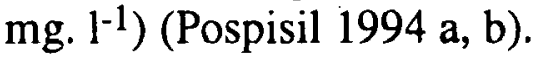

\subsection{Stations à Cladocères}

Les stations souterraines à échantillons positifs pour les Cladocères sont présentées sur les tableaux 1 et 2 et la figure 1. 
Tableau 1. Zone A du Lobau : stations souterraines à échantillons contenant des Cladocères. Pour la station LB (Freeze) voir les «Abréviations» Table 1. Zone A of Lobau : underground sites where positive samples of Cladocera were taken. For site LB (Freeze), see the «Abbreviations».

\begin{tabular}{|c|c|c|c|c|c|}
\hline Sonde & Prof. (m) & Date & Temp. $\left({ }^{\circ} \mathrm{C}\right)$ & Oxygène (mg. $\left.\mathrm{l}^{-1}\right)$ & Litres filtrés \\
\hline LA (2) & $0,4-0,5$ & 24.11 .1978 & - & - & 1 \\
\hline LA (3) & $0,4-0,5$ & 24.11 .1978 & - & - & 1 \\
\hline LA 1 & $0,4-0,5$ & 12.02 .1980 & 5 & 6 & 3 \\
\hline LB (1) & $0,4-0,5$ & 17.10 .1980 & 11 & 9 & 1 \\
\hline LB (2) & $0,4-0,5$ & 17.10 .1980 & 11 & 9 & 1 \\
\hline LB (3) & $0,4-0,5$ & 17.10 .1980 & 11 & 9 & 1 \\
\hline LB 1 & $0,4-0,5$ & 12.02 .1980 & 5 & 6,8 & 3 \\
\hline LB 3 & $0,4-0,5$ & 17.04 .1980 & 15 & 8,3 & 3 \\
\hline LC 1 & $0,4-0,5$ & 12.02 .1980 & 5 & 7,3 & 3 \\
\hline LC 1 & $0,4-0,5$ & 31.07 .1980 & - & 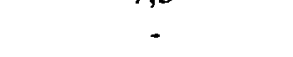 & - \\
\hline LC 2 & $0,4-0,5$ & 12.02 .1980 & 7 & 5,5 & 3 \\
\hline LA 40 (3) & $0,4-0,5$ & 24.11 .1978 & - & - & 1 \\
\hline LC 40 & $0,4-0,5$ & 23.08 .1978 & - & - & 3 \\
\hline LC 120 & 1,2 & 12.02 .1980 & - & - & 3 \\
\hline
\end{tabular}

Tableau 2. Zone $\mathrm{C}$ du Lobau : stations souterraines à échantillons contenant des Cladocères.

Table 2. Zone $\mathrm{C}$ of Lobau : underground sites where positive samples of Cladocera were taken.

\begin{tabular}{|c|c|c|c|c|c|}
\hline Sonde & Prof. (m) & Date & Temp. $\left({ }^{\circ} \mathrm{C}\right)$ & Oxygène (mg.|-1) & Litres filtrés \\
\hline D 3 & 2 & 28.05 .1991 & 13,3 & 1,5 & 5 \\
\hline D 3 & 4 & 16.01 .1992 & 3,1 & 4,3 & 5 \\
\hline D 3 & 4 & 15.04 .1993 & - & - & 5 \\
\hline D 3 & 6 & 15.04 .1993 & 8,4 & 4,2 & 5 \\
\hline D 3 & 7,5 & 16.01 .1992 & 3,2 & 4,0 & 5 \\
\hline D 4 & 2 & 16.01 .1992 & 2,4 & 4,7 & 5 \\
\hline D 4 & 4 & 16.01 .1992 & 2,5 & 4,5 & 5 \\
\hline D 4 & 5 & 16.01 .1992 & 2,5 & 4,5 & 5 \\
\hline D 9 & 4 & 19.01 .1993 & 0,6 & 6,1 & 5 \\
\hline D 10 & 1,5 & 28.05 .1991 & 12,3 & 4,2 & 5 \\
\hline D 10 & 5 & 28.05 .1991 & 11,7 & 1,1 & 5 \\
\hline D 15 & 10 & 16.01 .1992 & 4,5 & 1,1 & 5 \\
\hline R 1 & 1,7 & 11.09 .1992 & - & - & 5 \\
\hline R 1 & 1,7 & 14.04 .1993 & 10,1 & - & 5 \\
\hline $\mathrm{R} 2$ & 1,7 & 18.01 .1993 & 1,6 & - & 5 \\
\hline R 2 & 1,7 & 14.04 .1993 & - & - & 5 \\
\hline R $4 \mathrm{~d}$ & 2,8 & 14.04 .1993 & 0,1 & - & 5 \\
\hline R 8 & 1,8 & 14.04 .1993 & 0,7 & - & 5 \\
\hline T 3 & 2,5 & 20.10 .1992 & 16,8 & 0,1 & 5 \\
\hline
\end{tabular}

\subsection{Abréviations}

Stations à Cladocères (Fig. 1) :

$\mathrm{L}=$ Lobau; LA, LA1, LA40; LB, LB 1, LB3, LC1, LC2, LC40, LC120 = stations (sondes) à Cladocères. dans la zone $\mathrm{A}$;
LB $($ Freeze $)=$ les premiers $20 \mathrm{~cm}$ du sédiment, congelés avec de l'azote liquide, prélevés près de la sonde LB ;

OF = échantillon prélevé à l'interface eau-sédiment (au fond du bras mort Eberschüttwasser); 
(1), (2), (3) $=1^{\text {er }}, 2^{\text {eme }}$ et $3^{\text {eme }}$ litre d'un échantillon de 3 litres obtenu par filtrage, à l'aide de la pompe BouRouch ;

D3, D4, D9, D10, D15 = sondes à niveaux multiples $(\varnothing=5 \mathrm{~cm})$ dans la zone $C$;

$\mathrm{R} 1, \mathrm{R} 2, \mathrm{R} 4 \mathrm{~d}, \mathrm{R} 8, \mathrm{~T} 3=$ petites sondes $(\varnothing=2,5 \mathrm{~cm})$ dans la zone $C$.

Autres abréviations :

$\mathrm{SB}=$ espèce stygobionte $; \mathrm{SF}=$ stygophile $; \mathrm{SXR}=$ stygoxène régulière ; $S X=$ stygoxène;

ad. $=$ adulte $; \mathrm{B}=$ benthos $;$ éch. $=$ échantillon $;$ indiv. $=$ individu $;$ juv. $=$ juvénile $; \mathrm{N}=$ neuston $; \mathrm{ov} .=$ ovigère $; \mathrm{P}=$ plancton $;$ prof. $=$ profondeur $;$ temp. $=$ température $; \mathrm{V}=$ végétation.

\section{Résultats}

Nous avons identifié 16 espèces et sous-espèces de Cladocères dont 9 appartiennent à la famille des Chydoridae (Tableau 3). Pour chacun des 16 taxons, nous indiquons : le matériel étudié, l'aire de répartition, une brève caractérisation écologique (affinités pour les eaux stagnantes ou courantes, épigées ou souterraines), les catégories écologiques (pour les eaux épigées et souterraines) et d'éventuelles remarques.

Les données pour les eaux souterraines d'autres pays et les catégories écologiques sont extraites d'une synthèse inédite élaborée en 1993 par le premier auteur : «Aperçu sur les Cladocères des eaux souterraines du monde» ; ce manuscrit sera complété avec des données nouvelles par H. Dumont (Gent) et publié ultérieurement, en collaboration, dans une version anglaise.

Sididae (Baird, 1850).

Sida crystallina crystallina (O. F. Müller, 1776).

Matériel : D15, 10 m prof., 16.1.1992, 1 \% ad.

Paléarctique. Eaux stagnantes riches en végétation. Occasionnellement dans les eaux hyporhéiques (HautRhône français, jusqu'à -1 m) et phréatiques, mentionnée ici pour la première fois). Macrophytophile. SX?

Diaphanasosoma brachyurum (Lievin, 1848) emend. Negrea, 1983 (s. str.).

Matériel : LC40, 0,4 - 0,5 m prof., 23. 8. 1978, 3 \&q ad.

Paléarctique. Typique pour les eaux eutrophes profondes. Accidentellement dans la nappe alluviale du Lobau (c'est la première mention dans les eaux souterraines). Planctonique. SX?

Daphniidae (Straus, 1820).

Simocephalus vetulus (O. F. Müller, 1776).
Matériel : R1, 1,7 m. prof., 11. 9. 1992, 1. $\$$ ad.

Quasi cosmopolite. Eaux stagnantes et à courant lent, riches en végétation: Souvent dans les eaux souterraines d'Europe (notamment dans les bassins du Danube et du Rhône) : eaux des grottes, puits (jusqu'à - $7 \mathrm{~m}$ ), eaux hyporhériques (jusqu'à - $0,8 \mathrm{~m}$, parfois abondante), sources et maintenant dans la nappe alluviale du Lobau. Macrophytophile. SF.

Ceriodaphnia pulchella Sars, 1862.

Matériel : LC40, 0,4-0,5 m. prof., 23. 8. 1978, 1 \% ad.

Holarctique. Eaux stagnantes eutrophes riches en végétation, rivières et fleuves à courant lent. Occasionnellement dans la nappe alluviale du Lobau (première mention dans les eaux souterraines). Planctonique et macrophytophile. SX?

Il faut noter la présence dans deux stations épigées (LA. OF., 13. 6. 1978, 9 우 juv. et ad.; LE (2), 24. 11. 1978, 2 Ợ à éphippium; LA (3), 24. 11. 1978, 6 우 à éphippium) qui se trouvent dans la même zone du Lobau (A) mais à une autre période de l'année.

Scàpholeberis mucronata O.F. Müller, 1776.

Matériel : D3, 2 m. prof., 28. 5. 19912 \&o juv. et ad. (cornuta) ; D10, 1,5 m. prof. 28. 5. 1991, 1 \% ad. (cornuta) ; D10, 5 m. prof., 28. 5. 1991, 19 ad. (cornuta).

Paléarctique (et nord-néarctique?). Eaux stagnantes riches en végétation, rivières à courant lent. Parfois, dans les eaux hyporhéiques (Haut-Rhône français, jusqu'à - $0,9 \mathrm{~m}$ ) et phréatiques (seulement les découvertes mentionnées ici à -1,5-5 m) et dans les sources. Hyponeustonique et macrophytophile. SXR?

Remarque : les $\$ O$ de Lobau trouvées à $-1,5$ et $2 \mathrm{~m}$ avaient l'ocelle et l'oeil dépourvus de pigment ; celle trouvée à $-5 \mathrm{~m}$ avait du pigment mais celui-ci était en décomposition.

Ilyocryptidae Smirnov, 1992.

Ilyocryptus agilis Kurz, 1878.

Matériel : LB (1), 0,4 -0,5 m. prof., 17. 10. 1980, 1 \% ov.

Paléarctique. Eaux stagnantes, rivières et fleuves à courant lent. Parfois dans les eaux hyporhéiques (Haut Rhône français, jusqu'à - 1m) et dans la nappe alluviale du Lobau (seulement la découverte mentionnée ici). Pélophile et macrophytophile. SF?

Bosminidae (Baird, 1845).

Bosmina longirostris (O.F. Müller, 1785).

Matériel : D 3, 4 m prof., 16. 1. 1992, 1 \% ad. (similis) ; D3, 7,5 m prof., 16. 1. 1992, 4 \&ᄋ juv. et ad. (similis) ; D4, 2 m prof., 16. 1. 1992, 1 \& juv. (similis) ; D4, 4 m prof., 16. 1. 1992, 1 \% ad. (similis). 
Quasi cosmopolite. Eaux stagnantes, rivières et fleuves à courant lent. Souvent dans les eaux hyporhéiques (Haut-Rhône français jusqu'à - 0,6 m), phréatiques (puits d'Asie Centrale, nappe alluviale du Lobau jusqu'à - 7,5 m) et dans les grottes (Slovénie), dans des conduits d'eau (Roumanie). Planctonique et même macrophytophile. SF ?

Il faut remarquer la présence de cette espèce dans trois stations épigées : LA. OF., 13. 6. 1968, 1 \% juv. (similis); LB. OF., 23. 6. 1978, 1 \& ov. (typica); LE (3), 24. 11. 1978. 1 o ov. (pellucida), stations qui se trouvent:dans la zone A du Lobau. Il faut remarquer aussi que dans les eaux souterraines du Lobau, même en janvier, $B$. longirostris présente tous les stades de développement jusqu'à - $7 \mathrm{~m}$. Il est possible que les individus trouvés ici en janvier 1992 soient arrivés passivement par les horizons supérieurs de la nappe phréatique après les crues de décembre 1991 (en utilisant même les orifices des sondes piézométriques en plexiglas).

Chydoridae Stebbing, 1902.

Pleuroxus laevis Sars, 1862.

Matériel : LB3, 0,4-0,5 m prof., 17. 4. 1980, 1 qad.

Quasi cosmopolite. Eaux à fond limoneux, riches en végétation, rivières à courant lent. Souvent dans les eaux hyporhéiques (Haut-Rhône français, jusqu'à - 0,5 $\mathrm{m}$ ) et parfois dans celles des grottes (Slovénie) et phréatiques (nappe alluviale du Lobau). Pélophile et macrophytophile. SF?

\section{Alonella nana (Baird, 1843).}

Matériel : LB (Freeze), 0-0,2 m prof., 4.9.1980, 1 \% ad.; D3, 4 m prof., 15. 4. 1993, 19 ad.; D4, 5 m prof., 16. 1. 1992, 1 \% ad.; D9, $4 \mathrm{~m}$ prof., 19. 1. 1993., 1 \% ov.; R4 d, 2,8 m prof., 14. 4. 1993, 3 \&Q ov.; R8, 1,8 m prof., 14. 4. 1993, 11 \%ᄋ juv. et ov.

Holarctique. Eaux stagnantes et à courant lent. Assez fréquente dans les eaux hyporhéiques (Haut-Rhône français, jusqu'à - 0,6 m) et phréatiques (nappe alluviale du Lobau). Macrophytophile et pélophile. SF?

$A$ remarquer la présence de cette espèce dans quatre stations épigées : LA. OF., 13. 6. 1978, 5 \$o juv. et ad.; LB. OF., 23. 6. 1978, 27 \&ᄋ juv. et ov.; LC. OF., 23. 6. 1978, 1 \% ad.; LD. OF., 23. 6. 1978, 2 우 ad., stations qui se trouvent dans la zone A du Lobau. Dans les eaux souterraines de cet écosystème, elle est présente en janvier, avril et septembre, jusqu'à - 5 m (maximum 11 \$\&/ 51 à - $1,8 \mathrm{~m})$.

Chydorus sphaericus (O. F. Müller 1776) emend. Frey, 1980, (s. str.).

Matériel : D3, 6 m. prof., 15.4.1993, 1 q ad.; D9, 4. m prof., 19. 1. 1993, 3 \&Q ov.; D15, 10 m prof., 16. 1 .
1992, 1 \& ad.; R1, 1,7 m prof., 14. 4. 1993, 2 우아 ov.; R2, 1,7 m prof., 14. 4. 1993., 1 \% ov.

C. sphaericus s. str. est connu avec certitude seulement d'Europe. Eaux stagnantes, de préférence eutrophes, rivières et fleuves à courant lent. Fréquent et parfois abondant dans les eaux souterraines européennes (notamment dans les bassins du Danube, du Rhône et du Ter) : eaux des grottes, puits, eaux hyporhéiques (jusqu'à - $1 \mathrm{~m}$ ), citernes, conduits d'eau, filtres à sable, sources et maintenant dans la nappe alluviale du Lobau (voir ci-dessus). Macrophytophile, pélophile, psammophile et même planctonique. SF.

Dans l'écosystème du Lobau, l'espèce est présente en janvier et avril jusqu'à - $10 \mathrm{~m}$ (1 - 3 \&o ad./éch.).

Alona rectangula Sars, 1862.

Matériel : R2, 1,7 m prof., 18. 1. 1993, 1 \% ad.

Paléarctique, éthiopienne et orientale. Eaux stagnantes à fond limoneux, riches en détritus et végétation submergée, rivières et fleuves à courant lent. Fréquente et parfois abondante dans les eaux hyporhéiques (Haut-Rhône français, Ter etc, jusqu'à - $1 \mathrm{~m}$ ), dans les puits (Asie Centrale), grottes (Roumanie, Slovénie), réservoirs d'eau, filtres à sable, sources et maintenant dans la nappe alluviale du Lobau. Macrophytophile et pélophile. SF.

Alona guttata Sars, 1862.

Matériel : LA (2), 0,4-0,5 m prof., 24. 11. 1978, 7 \%ᄋ ov.; LA (3), 0,4-0,5 m prof., 24. 11. 1978., 10 \&Q juv. et ov.; LC1, 0,4-0,5 m prof., 31. 7. 1980, 1 \% ov.; LC2, 0,4-0,5 m prof., 12. 2. 1980, 1 \% ad.; LA 40 (3), 0,4-0,5 m prof., 24. 11. 1978, 19 ov.; T3, 2,5 m prof., 20. 10. 1992,2 \&ᄋ ad.

Quasi cosmopolite. Eaux stagnantes et à courant lent à limon et à végétation. Fréquente et parfois abondante (même dominante!) dans les eaux hyporhéiques (les bassins du Danube, du Rhône, du Ter etc, jusqu'à - 0,6 $\mathrm{m})$, dans les puits, filtres à sable et conduits d'eau (Roumanie), sources (Asie Centrale, Roumanie, Allemagne etc) et maintenant dans la nappe alluviale du Lobau. Macrophytophile et pélophile. SF.

Il faut aussi remarquer la présence d'Alona guttata dans trois stations épigées : LA. OF., 13. 6. 1978., 7 \%ᄋ juv. et ov.; LB. OF., 23. 6. 1978, 2 \%9 ov.; LE (2), 24. 11. 1978, 3 OO ov., stations qui se trouvent dans la zone A du Lobau. Dans les eaux souterraines, elle a une présence saisonnière presque constante (à l'exception du printemps), avec tous les stades de développement. L'ocelle des femelles adultes est plus petit ou à peu près égal à l'oeil.

Acroperus harpae (Baird, 1834). 
Matériel : LA (2), 0,4-0,5 m prof., 24. 11. 1978, 3 \%유 ov.; LA (3), 0,4-0,5 m prof., 24. 11. 1978, 4 \&ᄋ juv. et ov.; LA 1, 0,4-0,5 m prof., 12. 2. 1980, 1 \% ov.; LB (1), 0,4-0,5 m prof., 17. 10. 1980, 1 \& ad.; LB1, 0,4-0,5 m prof., 12. 2. 1980, 1 \& ov.; LB3, 0,4-0,5 m prof., 17. 4. 1980,8 \&ᄋ juv. et ov.; LC 120, 1,2 m prof., 12.2. 1980, 1 \% ad.

Holarctique, éthiopienne, orientale et néotropicale. Eaux stagnantes, riches en végétation, rivières et fleuves à courant lent. Fréquente mais pas du tout abondante dans les eaux hyporhéiques (Haut-Rhône français, jusqu'à - $0,6 \mathrm{~m}$ ), sources (Asie Centrale et Slovénie) et maintenant dans la nappe alluviale du Lobau. Macrophytophile et même pélophile. SF.

A remarquer la présence dans deux stations épigées de la zone A : LE (2), 24. 11. 1978, 1 \% ov.; L. OF., 12. 2. 1980, 1 \% ad. Dans les eaux souterraines du Lobau, cette espèce a une présence discontinue (février-avril et octobre-novembre) mais avec tous les stades de développement jusqu'à - 1,2 m.

Biapertura affinis (Leydig, 1860).

Matériel : LB (Freeze), 0-0,2 m prof., 4. 9. 1980, 1 \% ov.; LA (2), 0,4-0,5 m prof., 24. 11. 1978, 1 \% ad.; LA (3), 0,4-0,5 m prof., 24. 11. 1978, 1 \& ad.; LA1, 0,4-0,5 m prof., 12. 2. 1980,1 ㅇ ov.; LB (1), 0,4-0,5 m prof., 17. 10. 1980,1 \& juv.; LB (2), 0,4-0,5 m prof., 17. 10. 1980, 1 \& juv.; LB (3), 0,4-0,5 m prof., 17. 10. 1980, 1 $\$$ ad.; LB3, 0,4-0,5 m prof., 17. 4. 1980, 12 \&ᄋ juv. et ov.; LC1, 0,4-0,5 m prof., 12. 2. 1980., 4 \&o juv. et ov.; LC2, 0,4-0,5 m prof., 12. 2. 1980, 2 \$\% ov.; LC 120, 1,2 m prof., 12. 2. 1980, 1 \% ad.

Quasi cosmopolite. Eaux stagnantes et rivières à courant lent avec limon, sable et détritus sur le fond. Fréquente et abondante dans les eaux hyporhéiques (bassins de la Sava et du Rhône, jusqu'à - $0,6 \mathrm{~m}$ ), dans des grottes (Slovénie, Irlande), des sources karstiques (Slovénie) et maintenant dans lä nappe alluviale du Lobau. Psammo-détrito-pélo- et macrophytophile. SF.

Il faut remarquer la présence dans quatre stations épigées situées dans la zone A : LB. OF., 23. 6. 1978, 29 ф̊q juv. et ov.; LC. OF., 23. 6. 1978, 1 \% juv.; LE (2), 24. 11. 1978, 1 \% ov.; L. OF., 12. 2. 1980, 1 \% ad. Dans les eaux souterraines du Lobau, cette espèce a une présence saisonnière presque continuelle (à l'exception de l'été quand elle est présente seulement à la surface), mais avec tous les stades de développement, jusqu'à $1,2 \mathrm{~m}$.

\section{Rhynchotalona falcata (Sars, 1862)}

Matériel : LB3, 0,4-0,5 m prof., 17. 4. 1980, 1 \& ad.

Nord-holarctique. Eaux stagnantes à fond sabloneux et pierreux à détritus. Mentionnée deux fois seulement dans les eaux souterraines : l'interstitiel d'un lac de cratère à Wales (Dumont, comm. pers.) et maintenant dans la nappe alluviale du Lobau. Psammo- et lithophile. SX.

A remarquer la présence dans deux stations épigées, situées dans la zone A : LA. OF., 13. 6. 1978, 1 ad.; LB. OF., 23. 6. 1978, 1 \% ad.

Les trois femelles trouvées sont non ovigères et ont l'ocelle plus grand que l'oeil (et non de la même taille).

\section{Monospilus dispar Sars, 1862.}

Matériel : LA 40 (3), 0,4-0,5 m prof., 24. 11. 1978, 1 o ad.

Holarctique et éthiopienne. Eaux stagnantes et à courant lent avec sable ou limon et détritus sur le fond. Fréquente mais pas abondante dans les eaux hyporhéiques (Haut-Rhône français, jusqu'à - $1 \mathrm{~m}$ ), puits (Asie Centrale) et maintenant dans la nappe alluviale du Lobau. Psammo- et pélophile. SF ?

Il faut remarquer la présence dans une station épigée de la même zone aquatique (A) : LB. OF., 23. 6. 1978, 3 우 ov.

\section{Discussion}

La présence des Cladocères dans les milieux aquatiques souterrains a été longtemps considérée fortuite. En l'absence d'espèces stygobies, les captures étaient interprétées comme l'effet de migrations passives, accidentelles, dans les eaux hypogées. Mais l'assertion que les Cladocères sont incapables de mener une vie exclusivement souterraine - en formant des peuplements permanents - a été infirmée par la découverte de certaines espèces qui présentent des caractères régressifs d'adaptation. Il s'agit de cinq espèces d'Alona, soit dépourvues d'oeil et d'ocelle, soit seulement d'oeil ou ayant les deux évidemment réduits, qui sont certainement stygobies : A. hercegovinae et $A$. sketi des grottes du karst dinarique (Brancelj 1990,1992, Brancelj \& Sket 1990), A. smirnovi des eaux interstitielles du lac Ochrid (Petkovski \& Flössner 1972), $A$. phreatica découverte dans les eaux interstitielles d'une rivière d'Auvergne (Dumont 1983) et A. alsafadii des eaux interstitielles d'une rivière du Yemen (Dumont $\&$ Brancelj 1994).

On connaît dans le monde environ 450 espèces de Cladocères, dont environ $90(20 \%)$ peuvent vivre aussi dans les milieux hypogés (Motas et al. 1962, Negrea 1983, 1994). Parmi celles-ci, plus de 60 espèces ont été recueillies dans des eaux hyporhéiques (rhithro- et potamostygal sensu Botosaneanu 1986 et hyporhéal sensu Danielopol 1980), environ 40 espèces dans des 
puits ou extraites à l'aide de sondes (eustygal), presque 30 dans les eaux stagnantes de grottes (karstostygal), 13 dans des conduits d'eau, filtres à sable, citernes et réservoirs d'eau, plus de 30 dans différentes sources et collecteurs de sources, 7 dans les eaux interstitielles de lacs (limnostygal) et 1 dans des rivières de grottes (troglorhithrostygal) (Negrea 1994).

Dans la nappe alluviale du Lobau (Vienne) nous n'avons pas trouvé d'espèces totalement inféodées aux habitats souterrains, mais nous avons mis en évidence - pour la première fois - cinq espèces stygophiles parmi les 16 taxons : Alonella nana, Chydorus sphaericus s. str., Alona guttata, Acroperus harpae et Biapertura affinis (Tableau 3). Ces espèces appartiennent à la famille des Chydoridae, connue par ses représentants détritivores benthiques et macrophytophiles, généralement mauvais nageurs mais bons rampants et qui peuvent coloniser les milieux hypogés.

\subsection{Remarques biogéographiques}

Les 16 espèces stygophiles et stygoxènes du Lobau (Tableau 3) appartiennent à six catégories zoogéographiques. Quatre espèces sont paléarctiques (Sida crystallina crystallina, Diaphanosoma brachyurum s. str., Scapholeberis mucronata et Ilyocryptus agilis), une paléarctique, éthiopienne et orientale (Alona rectangula), trois holarctiques (Ceriodaphnia pulchella, Alonella nana et Rhynchotalona falcata), une holarctique et éthiopienne (Monospilus dispar), une holarctique, éthiopienne, orientale et néotropicale (Acroperus harpae) et cinq quasi cosmopolites (Simocephalus vetulus, Bosmina longirostris, Pleuroxus laevis, Alona guttata et Biapertura affinis). Quant à l'espèce Chydorus sphaericus s. str., redécrite par Frey en 1980, elle est connue avec certitude d'Europe seulement.

On remarque l'absence d'espèces' endémiques ou à répartition européenne et la présence de taxons à vaste aire de répartition et à valence écologique plus ou moins grande. Ceux-ci sont résistants aux fluctuations des facteurs limitatifs qui caractérisent cet écosystème souterrain pendant les crues du Danube et peuvent supporter l'hypoxie pendant les périodes entre deux crues.

\subsection{Remarques écologiques}

4.2.1. Espèces caractéristiques de la nappe alluviale du Lobau

Les cinq espèces stygophiles de l'écosystème souterrain du Lobau ont été caractérisées par leur fréquence, leur abondance relative (nombre d'individus) et par

\begin{tabular}{|c|c|c|c|c|c|c|c|c|c|c|c|c|c|c|c|c|c|}
\hline \multirow[t]{3}{*}{ Famille } & \multirow[t]{3}{*}{ Espèce. } & \multirow{3}{*}{$\begin{array}{l}\mathrm{N}^{\circ} \text { éch. } \\
\text { positifs }\end{array}$} & \multirow{3}{*}{$\begin{array}{l}N^{\circ} \text { total } \\
\text { indiv. }\end{array}$} & \multicolumn{12}{|c|}{$\mathrm{N}^{\circ}$ des individus (prof. en $\mathrm{m}$ ) } & \multicolumn{2}{|c|}{ Pour eaux Pour eaux } \\
\hline & & & & \multicolumn{3}{|c|}{ Zone A } & \multicolumn{9}{|c|}{ Zone C } & & \\
\hline & & & & $0-0,2$ & $0,4-0,5$ & 1,2 & 1,5 & $1,7-1,8$ & 2 & $2,5-2,8$ & 4 & 5 & 6 & 7,5 & 10 & & \\
\hline \multirow[t]{2}{*}{ Sididae } & Sida crystallina & 1 & 1 & - & - & - & - & - & - & - & - & - & - & - & 1 & $\mathbf{v}$ & $s \times ?$ \\
\hline & Diaphanosoma brachyunum & 1 & 3 & - & 3 & - & - & - & - & - & - & - & - & - & - & $\mathbf{P}$ & $\mathrm{sx} ?$ \\
\hline \multirow[t]{3}{*}{ Daphniidae } & Simocephalus vetulus & 1 & 1 & - & - & - & - & 1 & - & - & - & - & - & - & - & $v$ & $\mathrm{SF}$ \\
\hline & Ceriodaphnia pulchella & 1 & 1 & - & 1 & - & - & - & - & $:$ & - & - & - & - & - & $p+V$ & sX? \\
\hline & Scapholeberis mucronata & 3 & 4 & - & - & - & 1 & - & 2 & - & - & 1 & - & - & - & $\mathrm{V}+\mathrm{N}$ & $\mathrm{sX} ?$ \\
\hline Ilyocryptidae & Ilyocryptus agilis & 1 & 1 & - & 1 & - & - & . & - & - & - & - & - & - & - & $B+V$ & SF ? \\
\hline Bosminidae & Bosmina longirastris & 4 & 7 & - & - & - & - & . & 1 & - & 2 & - & - & 4 & - & $\mathbf{P}$ & $\mathrm{SF} ?$ \\
\hline \multirow[t]{9}{*}{ Chydoridae } & Pleuroxus laevis & 1 & 1 & - & 1 & - & - & - & - & - & - & . & - & - & - & $\mathbf{V}+\mathbf{B}$ & $\mathrm{SF} ?$ \\
\hline & * Alonella nana & 6 & 18 & 1 & - & - & . & 11 & - & 3 & 2 & 1 & - & - & - & $V+B$ & SF ? \\
\hline & * Chydorus sphaericus & 5 & 8 & - & - & - & - & 3 & - & - & 3 & - & 1 & - & 1 & $V+B$ & $\mathbf{S F}$ \\
\hline & Alona rectangula & 1 & 1 & - & - & - & - & 1 & - & - & - & - & $:$ & - & - & $V+B$ & SF \\
\hline & *Alona guttata & 6 & 22 & - & 20 & - & - & - & - & 2 & - & - & - & $\cdot$. & - & $V+B$ & $\mathbf{S F}$ \\
\hline & * Acropenus harpae & 7 & 19 & - & 18 & 1 & - & -. & - & - & - & . & - & - & - & $v$ & $\mathbf{S F}$ \\
\hline & * Biapertura affinis & 11 & 26 & 1 & 24 & 1 & - & . $\cdot$ & - & - & - & - & - & - & - & $B+V$ & $\mathbf{S F}$ \\
\hline & Rhynchotalona falcata & 1 & 1 & - & 1 & - & . & - & - & - & - & - & - & - & - & B & $\mathbf{s X}$ \\
\hline & Monospilus dispar & 1 & 1 & - & 1 & - & - & - & - & - & - & - & - & - & - & B & SF ? \\
\hline Total & 16 & 51 & 115 & 2 & 70 & 2 & 1 & 16 & 3 & 5 & 7 & 2 & 1 & 4 & 2 & - & - \\
\hline
\end{tabular}

Tableau 3. Structure de l'association de Cladocères de la nappe alluviale du Lobau. * Espèces caractéristiques. Table 3. Structure of the Cladocera association from the alluvial stratum of Lobau. ${ }^{*}$ Characteristic species. 
leur présence à différentes profondeurs dans les zones $A$ et $C$ pendant le prélèvement saisonnier des échantillons (Tableaux 1, 2, 3).

Alonella nana est fréquente surtout dans la zone $\mathrm{C}$ en hiver, au printemps et à l'automne (en été aussi, mais seulement dans le benthos de la zone $\mathrm{A}$, donc à la surface) et relativement abondante en avril (maximum 11 $\$$ juv. et ov. $/ 5 \mathrm{l}$ à $1,8 \mathrm{~m}$ prof.). Etant très petits $(0,22-0,26 \mathrm{~mm})$ et mauvais nageurs, les individus préfèrent se trainer dans les interstices du substratum. De ce fait, il est possible qu'il existe dans la zone $C$ une population souterraine quasi permanente jusqu'à - $5 \mathrm{~m}$.

Chydorus sphaericus s. str. est présent seulement dans la zone $\mathrm{C}$ en hiver et au printemps, en petit nombre (maximum 3 QO/5 1 à 1,7 et $4 \mathrm{~m}$ prof.). Cette espèce peut se trainer, s'enfoncer dans le substratum et en même temps bien nager. Il est possible qu'il s'agisse d'une population souterraine étant donné qu'il a été trouvé des femelles ovigères jusqu'à - $4 \mathrm{~m}$, non ovigères jusqu'à - $10 \mathrm{~m}$, mais aucune femelle dans le benthos d'Eberschüttwasser!

Alona guttata est présente dans toute la zone A en hiver, été et automne, et dans le benthos d'Eberschüttwasser en été et automne seulement. Le maximum d'individus a été obtenu en novembre ( 7 우 ov./1 1 à 0,4-0,5 m prof., dans la zone A). C'est une bonne rampante et une mauvaise nageuse qui a été trouvée jusqu'à - 2,5 m (sonde T3). Il est possible que dans la zone A existe une population qui, pendant l'hiver, se trouve seulement dans le milieu souterrain.

Acroperus harpae est présente seulement dans la zone A jusqu'à - 1,2 m en hiver, printemps et automne et dans le benthos d'Eberschüttwasser en hiver et automne. Le maximum d'individus a été observé en avril ( 8 $\$$ juv. et ov./3 1 à $0,4-0,5 \mathrm{~m}$ prof.). Il est difficile de comprendre l'absence de cette espèce macrophytophile et pélophile pendant la saison chaude (mai-septembre) dans la nappe alluviale; il faut remarquer cependant sa présence au printemps (avril) seulement dans la nappe alluviale.

Biapertura affinis, espèce benthique par excellence, est présente dans beaucoup de stations jusqu'à - $1,2 \mathrm{~m}$, toutes dans la zone A, en hiver, printemps et automne et dans le benthos d'Eberschüttwasser en hiver, été et automne. Le maximum d'individus a été observé en Avril (12 Oo juv. et ov./3 1 à 0,4-0,5 m prof.). Il s'agit d'une population qui pendant la saison froide (automne et hiver) occupe les deux biotopes mais qui, au printemps, se trouve seulement dans la nappe alluviale et pendant l'été, quand l'eau souterraine devient hypoxique, seulement dans le benthos.
4.2.2. Structure de l'association de Cladocères et changements saisonniers.

L'association des Cladocères de la nappe alluviale du Lobau comprend 10 espèces dans la zone $A$ et 8 dans la zone $C$, avec seulement deux espèces communes, toutes les deux stygophiles et caractéristiques de cette association : Alonella nana et Alona guttata (Tableau 3). Ainsi, comme il résulte de l'analyse faite $\S 4.4 .1$, les cinq espèces caractéristiques sont toujours présentes pendant la saison froide, depuis octobre-novembre jusqu'en avril (à l'exception de Chydorus sphaericus en automne, et d'Alona guttata au printemps) et absentes pendant la saison chaude de mai à septembre (à l'exception d'Alona guttata, l'été). Le phénomène peut être expliqué par les conditions hydrologiques (voir aussi $\S 2.2$ ).

Surtout dans la zone $C$, pendant la saison froide, l'eau du bras mort Eberschüttwasser, plus froide et riche en oxygène, pénètre dans l'eau souterraine et assure pendant tout l'hiver la vie des espèces de l'association souterraine de Cladocères, 1'apparition des $0^{\prime} \sigma^{\circ}$ et des $\$ q$ gamogénétiques n'étant pas nécessaire. Dans le même temps, l'eau qui reste à la surface, devenant trop froide, provoque la disparition de la majorité des espèces, qui réapparaitront au printemps par l'intermédiaire des éphippiums.

L'association de Cladocères de la nappe alluviale du Lobau contient également trois espèces planctoniques : Diaphanosoma brachyurum et Ceriodaphnia pulchella, chacune trouvée une seule fois, en août, dans la station LC 40 de la zone A (Tableaux 1 et 3) et Bosmina longirostris qui a été aussi trouvée une seule fois, mais en janvier dans les stations D3 et D4 de la zone C à -2-2,7 $\mathrm{m}$, toujours représentée par des juvéniles et par des femelles sans oeufs (Tableau 1 et 3). Ces espèces sont probablement accidentelles pour l'association souterraine de Cladocères.

La répartition spatiale des Cladocères dans la nappe alluviale du Lobau indique que ceux-ci se trouvent habituellement dans les zones en liaison hydrologique étroite avec le bras mort Eberschüttwasser. C'est le cas de toutes les sondes de la zone $\mathrm{A}$, ainsi que des sondes de la zone C (R1, R2, D3, D4, R4) situées à la limite «roseau-forêt» qui forme fréquemment la ligne du bord de l'Eberschüttwasser et des sondes situées dans le voisinage, comme $\mathrm{R} 8$ (qui, ainsi que $\mathrm{R} 1$, présente des valeurs d'oxygène élevées) (Mosslacher 1994, Pospisil 1994 b) et D9 (où d'autres animaux de surface ont été aussi trouvés). Toutes ces données montrent que les espèces benthiques de l'eau souterraine du Lobau sont en liaison étroite avec celles de surface, formant des populations communes «nappe alluviale- 
benthos», dont la dynamique est fortement influencée par le régime hydrologique.

\section{Conclusion}

Cette étude des Cladocères souterrains des bras morts du Danube a montré que, en étudiant les conditions hydrologiques, il faut tenir compte de l'influence des eaux de surface sur les eaux souterraines, la présence des Cladocères constituant alors un indicateur utile.

Nos résultats pourraient être un point de départ et inciter d'autres chercheurs à approfondir cette étude qui nécessiterait un effort semblable d'échantillonnage sur toute la longueur du Danube avant que d'autres espèces ne disparaissent sous l'effet de la pollution.

\section{Remerciements}

Nous remercions M. le Prof. Dan L. Danielopol (Mondsce, Autriche) pour le matériel de Cladocères, son aide bibliographique, ainsi que pour la lecture critique du manuscrit.

Pour la période 1991-1993, l'échantillonnage des Cladocères a été réalisé avec l'aide matérielle du «Fonds für Forderung der wissenschaftlichen Forschung», Vienne (Projet P 7881, attribué à D.L. Danielopol).

\section{Travaux cités}

Botosaneanu L. (ed.). 1986. - Stygofauna mundi. E.J. Brill/Dr. Backhuys, Leiden : $750 \mathrm{p}$.

Brancelj A. 1990. - Alona hercegovinae n.sp. (Cladocera : Chydoridae), a blind cave-inhabiting Cladocera from Hercegovina (Yugoslavia). Hydrobiologia, 199 : 7-16.

Brancelj A. 1992. - Alona sketi sp.n. (Cladocera : Chydoridae), the second cave-inhabiting cladoceran from Yugoslavia. Hydrobiologia, 248 : 105-114.

Brancelj A. \& Sket B. 1990. - Occurrence of Cladocera (Crustacea) in subterranean waters in Yugoslavia. Hydrobiologia, 199 : 7-16.

Danielopol D.L. 1976 a. - The distribution of the fauna in the interstitial habitats of riverine sediments of the Danube and the Piesting (Austria). Int. J. Speleol., $8: 23-51$.

Danielopol D.L. 1976 b. - Zoogeographische Probleme der Grundwasserfauna der Donau und ihrer Zuflüsse in Österreich. Sitzungsberichte Österr. Akad. Wiss. Math.-naturw. Kl., 13 : 1-6.

Danielopol D.L. 1976 c. - Sur la distribution géographique de la faune interstitielle du Danube et de certains de ses affluents en Basse Autriche. Int. J. Speleol., 8 : 323-329.

Danielopol D.L. 1980. - The role of the limnologist in groundwater studies. Int. Rev. ges. Hydrobiol., 65 (6) : 777-791.

Danielopol D.L. 1983. - Der Einfluss organischer Verschmutzung auf das Grundwasser-Ökosystem der Donau im Raum Wien und Niederösterreich. Beiträge Gewässerökologie, Bundesministerium für Gesundheit und Umweltschutz (Hrsg.), 5/83 : 1-155.

Danielopol D.L. 1984. - Ecological investigations on the alluvial sediments of the Danube in the Vienne area - a phreatobiological project. Verh. Internat. Verein. Limnol., 22 : 1755-1761.

Danielopol D.L. 1989. - Groundwater fauna associated with riverine aquifers. J. N. Am. Benthol. Soc., 8 : 18-35.

Danielopol D.L. 1991. - Spatial distribution and dispersal of interstitial Crustacea in alluvial sediments of a backwater of the Danube at Vienna. Stygologia, $6: 97-110$.
Danielopol D.L., Dreher J.; Gunatilaka A., Kaiser M., Niederreiter R., Pospisil P., Creuzé des Chatelliers M. \& Richter A. 1992. - Ecology of organisms living in a hypoxic groundwater environment at Vienna (Austria); methodological questions and preliminary results. First. Int. Conf. Goundw. U.S. Env. prot. Agency, Amer. Water Res. Assoc. : 79-90.

Danielopol D.L. \& Niederreiter R. 1987. - A sampling device for groundwater organisms and oxygen measurement in multi-level monitoring wells. Stygologia, $3: 252-263$.

Dreher J., Pospisil P. \& Danielopol D.L. 1995. - The role of hy. drology in defining a groundwater ecosystem within the wet. land of the Danube at Vienna. Cambridge Univ. Press, Cambridge (sous presse).

Dumont H.J. 1983. - Discovery of groundwater-inhabiting Chydoridae (Crustacea : Cladocera), with the description of two new species. Hydrobiologia, $106: 97-106$.

Dumont H.J. \& Brancelj A. 1994. - Alona alsafadii n.sp. from Yemen, a primitive, groundwater-dwelling member of the $A$. karua group. Hydrobiologia, $281: 57-64$.

Gibert J., Ginet R. \& Reygrobellet J.L. 1981. - Structure et fonctionnement des écosystèmes du Haut-Rhône français. 9 : Analyse des peuplements de deux stations phréatiques alimentant des bras morts. Int. J. Speleol., $11: 141-158$.

Haase M. 1993. - Hauffenia kerschneri (Zimmermann 1930) : zwei Arten. zweier Gattungen (Caenogastropoda : Hydrobiidae). Arch. Moll., 121 (1/6) : 91-109.

Klausnitzer B. \& Pospisil P. 1991. - Larvae of Cyphon sp. (Coleoptera, Helodidae) in Ground Water. Aquatic Insects, 13, 3 : 161-166.

Mosslachert F. 1994. - Beobachtungen eines Grundwasserökosystems in Freiland (Lobau, Wien) und experimentelle Untersuchungen über die Respirometrie, Fress-und Bewegungsaktivität einiger Süsswasserisopoden (Crustacea, Asellidae). Diplomarbeit, Univ. Wien : 80 p.

Motas C., Botosaneanu L. \& Negrea S. 1962. - Cercetari asupra biologiei izvoarelor si apelor freatice din partea centrala a Campiei Romane. Edit. Academiei, Bucuresti : 366 p.

Negrea S. 1983. - Cladocera. In Fauna R.S. Romania; Edit. Academiei, Bucuresti, 4 (12) : 1-399.

Negrea S. 1994. - Cladocera. In Encyclopaedia Biospeologica, Impr. Fabbro, Saint-Girons (France), 1 :99-104.

Niederreiter R. \& Danielopol D.L. 1991. - The use of mini-videocameras for the description of groundwater habitats. Mitteilungsbl. d. hydrographischen Dienstes in Österreich, 65/66 : 85-89.

Petkovski T. \& Flössner D. 1972. - Eine neue Alona-Art (Crustacea : Cladocera) aus dem Ochridsee. Fragm. Balcan. (Skopje), $9: 97-106$

Pospisil P. 1989. - Acanthocyclops gmeineri n.sp. (Crustacea, Copepoda) aus dem Grundwasser von Wien (Österreich) : Bemerkungen zur Zoogeographie und zur Sauerstoffsituation des Grundwassers am Fundort. Zool. Anz., 223 (3/4) : 220-230.

Pospisil P. 1994 a. - Die Grundwassercyclopiden (Crustacea, Copepoda) der Lobau in Wien (Österreich)-faunistische, taxonomische und ökologische Untersuchungen. Diss. Univ. Wien : 222 p.

Pospisil P. 1994 b. - The groundwater fauna of a Danube aquifer in the wetland Lobau in Vienna, Austria. In : Groundwater Ecology J. Gibert, D.L. Danielopol \& J. Stanford eds., Academic Press, San Diego : 347-366.

Pospisil P. \& Danielopol D.L. 1990 - Vorschläge für den Schutz der Grundwasserfauna im geplanten Nationalpark «Donauauen» östlich von Wien, Österreich. Stygologia, 5 (2) : 75-85.

Rogulj B., Danielopol D.L., Marmonier P. \& Pospisil P. 1993. Adaptative morphology, biogegraphical distribution and ecology of the species group Mixtacandona hvarensis (Ostracode, Candoninae). Mem. Biospéol., 20 : 195-207. 